\title{
Nodal Peripheral T-cell Lymphoma with T Follicular Helper Phenotype Present CD20 Positive: A Case Report and Review of Literature
}

\section{jianxian huang}

Sun Yat-sen University First Affiliated Hospital

Ni Liu

Sun Yat-sen University First Affiliated Hospital

wenhui zhang

Sun Yat-sen University First Affiliated Hospital

fang liu

Southern Medical University Nanfang Hospital

Qinghua Cao ( $\nabla$ caoqhua@mail.sysu.edu.cn)

Sun Yat-sen University First Affiliated Hospital https://orcid.org/0000-0002-1405-040X

\section{Case Report}

Keywords: peripheral T-cell lymphoma, T-follicular helper phenotype, CD20 positive, case report

Posted Date: September 27th, 2021

DOI: https://doi.org/10.21203/rs.3.rs-917471/v1

License: (c) (1) This work is licensed under a Creative Commons Attribution 4.0 International License. Read Full License 


\section{Abstract}

Background: T follicular helper cell is considered to be involved in B cell and a subset of effector T cell maturing. Nodal peripheral T-cell lymphoma with T-follicular helper phenotype (NPTCL-TFH) is a subset of peripheral T-cell lymphoma defined by expression of at least 2 or 3 TFH markers. Here we report a case of typical NPTCL-TFH while the B cell marker CD20 is positive.

Case presentation: A 62-year-old male presented to our hospital with a symptom of dizzy and neck mass for 10 days. Physical examination and PET/CT revealed right cervical, armpit and inguinal lymphadenopathy, some of which were accompanied by pain. In the nodule biopsy, lymph node structure is destroyed, completely effaced by intermediate-sized monotonous lymphoid cells with round nuclei and abundant mitosis. Immunohistochemical staining confirmed that tumor cells were positive for $\mathrm{T}$ cell marker, and expressed several markers of TFH (CD4, Bcl-6, CXCL-13 and PD-1 ). Besides, in situ hybridization of EB virus showed scattered positive and TCR gamma arrangement is positive. However, with all these evidences of NPTCL-TFH, the tissue weirdly expressed CD20, a B lymphocyte maturing marker. The patient gave up treatment and unfortunately was died of tumor cachexia after 4-5 months.

Conclusions: We report the first case of CD20 positive in a NPTCL-TFH. More investigation and knowledge of this new phenotype need to be explored.

\section{Background}

Nodal peripheral T-cell lymphoma (NPTCL) is a rare type of lymphoma, accounts for about 7\% of nonHodgkin lymphoma (NHL)[1]. It has a broad morphologic spectrum with significant overlap to other lymphoma[2]. As for a new umbrella category of PTCL, nodal peripheral T-cell lymphoma with T-follicular helper phenotype (NPTCL-TFH) was firstly classified in the 2017 revision of the World Health Organization (WHO) classification of hematolymphoid neoplasms. Its characteristic feature is a predominant, dense, expanded interfollicular infiltrate of small- and medium-sized lymphoid cells admixed with a few large cells, possibly mimicking angioimmunoblastic T cell lymphoma(AITL)[3]. The difference between them is that TFH phenotype is defined by expression of at least 2 or 3 TFH markers such as CD4, PD-1, CD10, BCL6, CXCL13, and ICOS[4, 5].

CD20 is a well-known B lymphocyte-specific membrane protein and is important in mounting optimal humoral immunity[6]. Its expression is associated with increased B cell signaling and T cell-dependent immune responses[7]. T-cell lymphoma rarely express CD20 unless there are special circumstances like T cell-independent immune responses[8, 9]. So far there is no literature of CD20 expressed on NPTCL-TFH.

We report here a case of NPTCL-TFH with aberrant CD20 expression, hoping to provide cases and more cognition on this new type of peripheral T-cell lymphoma.

\section{Case Presentation}


The patient was a 62-year-old male who presented dizziness for 10 days without special cause. A week before admission, he found a mass on his right neck. Additional evaluation showed his right cervical, armpit and inguinal lymphadenopathy, some accompanied by pain. B symptom like fever, drenching night sweats, weight loss were not seen. The patient informed about smoking and drinking for 40 years, and his general medical history revealed no specific surgery or disease. He also denies the family cancer history.

At the time of consultation, the patients' blood routine examination revealed that hemoglobin was 177 $\mathrm{g} / \mathrm{L}$, White blood cells, Platelets, Lactate dehydrogenase and Uric acid were within the reference range, no abnormality in other blood biochemistry tests was observed. Positron emission tomography / computed tomography (PET/CT) were applied to value multiple lymphadenopathies of the patient, including right jaw, bilateral cervical, right supraclavicular area, bilateral armpit, bilateral chest muscle, hilar, bilateral internal mammary, inferior vena cava on the diaphragm, etc. Result showed the size of the largest lymph node was about 3.4 centimeter in abdomen (Fig. 1). The shadow of swollen lymph node was round, cystic and low density.

Histological examination were made to figure out its properties. After the biopsy of the mass of his right neck, we got a pile of broken white tissue. Hematoxylin and eosin-stained sections showed the lymph node structure was destroyed, completely effaced by intermediate-sized monotonous lymphoid cells (Fig. 2). Otherwise, atypical lymphocytes were medium in size, with irregular nuclei, some of which could be seen with nucleoli, and the cytoplasm of the cells was rich and lightly stained; a small number of cells were large in size and looked like immune maternal or central maternal; mitosis were easy to see. As for the cell background, there were no eosinophils, neutrophils or Reed-Sternberg-like cells. The blood vessels of the interstitium were obviously proliferated, but high endothelial venule(HEV) hyperplasia is not obvious. All of the features pointed to the haematopoietic and lymphoid tumor. Unlike angioimmunoblastic T-cell lymphoma, it did not show a prominent polymorphic inflammatory background, vascular proliferation, or expansion of follicular dendritic cell meshworks.

To figure out the diagnosis, we did immunohistochemical staining of a set of markers. The results suggest that T cell markers such as CD3, CD5, CD2 and CD4 were positive. Detection of CD7, CD8, ALK, CD30, CD10 were negative. What interest us is that, CD20, the marker often related with $B$ cell maturing, also present positive here. But CD19, PAX-5, CD79a of whom suggest B-cell lymphoma were negative. IHC staining of this lymphoma sample confirmed CD4, Bcl-6, PD-1 and CXCL-13 positive. In the meantime, CD21 and CD23 clearly showed no follicular dendritic cell expansion, which support our diagnose of NPTCL-TFH. Additionally, tumore cells were negative for cyclinD1, IgD, MUM1, TdT, C-myc etc. Moreover, in situ hybridization reveals scattered positive EBER cells. The positive of clonal T-cell receptor (TCR) rearrangements further supported the diagnosis of $\mathrm{T}$ cell lymphoma. Analyze of $\mathrm{IgH}$ rearrangement is negative. In this regard, our final diagnosis is peripheral T-cell lymphoma with T follicular helper phenotype (Fig. 3). Unfortunately, the patient gave up treatment for financial reasons, and died of severe cancer cachexia 4-5 months after the biopsy. No autopsy was performed. 


\section{Discussion}

T follicular helper cell (TFH) is a type of CD4 + helper T cell subset, located in the follicular germinal center to promote B cell expansion and differentiation[10]. The 2008 World Health Organization (WHO) Classification scheme broadly grouped PTCLs into Angioimmunoblastic T-cell lymphoma (AITL) and PTCL, not otherwise specified (NOS). At that time lymphoma of $\mathrm{T}$ follicular helper cell origin(TFH-NL) was belong to PTCL, NOS. However, the 2017 World Health Organization (WHO) Classification scheme turned to grouped PTCLs into TFH-NL and PTCL, NOS. Currently identified categories of T-follicular helper cellderived lymphomas include AITL, follicular T-cell lymphoma (FTL) (equivalent to the follicular variant of the original peripheral T), and NPTCL-TFH[1, 11]. The diagnostic criteria of NPTCL-TFH are: a) shared immunophenotypic features with AITL; b) expressed at least 2(ideally 3) TFH markers; c) lack of Inflammatory/polymorphous background; d) few HEV proliferation; e) no follicular dendritic cell(FDC) meshwork expansion[12]. In a review by Parwiz $\mathrm{J}$ et al., $\mathrm{T}$ - and NK-cell lymphomas comprise only about $6 \%$ of all lymphoproliferative disorders, and the most frequent type is the not otherwise specified (NOS) category[13], NPTCL-TFH only account for less than $1 \%$ of all T-cell neoplasms[14, 15], therefore our case is very rare. Although our case is not the first NPTCL-TFH case reported, we firstly found CD20 expressed in this disease. All of the morphologic features, immunohistochemical results, scattered EBV + cells and Tcell arrangement positive proved our case to be a NPTCL-TFH. What interest us is CD20 get strong staining on the tissue. As a recognized B cell marker, CD20 is less well positive on T cells. The first case of CD20-positive peripheral T-cell lymphoma was reported by Yokose et al. in 2000,[16] then CD20positive AITL by Tachibana et al. in 2011[17]. Recently there were more cases observed in NPTCL[18, 19], however we report the very first case presented CD20 positive in NPTCL-TFH after 2017 WHO Classification scheme. CD20 positivity in T-cell lymphomas may include derivation from subsets of CD20 positive T-cells undergoing neoplastic transformation or CD20-acquisition following neoplastic transformation of the T-cells[19]. We suppose that T-follicular helper cells may have a supporting effect on B cells in the germinal center, and thus differentiate into CD20-expressing cells. Or TFH cells have uncertain direction of differentiation of progenitor $B$ cells.[20]

NPTCL-TFH should be distinguished from other types of peripheral T cell lymphomas, especially AITL. The clinical difference between AITL and NPTCL-TFH remains unclear, for they both have the symptoms of multiple lymphadenopathies, splenomegaly, B symptoms, hypergammaglobulinemia, eosinophilia, or a positive Coombs test[14]. In regards of pathology, AITL are disorganized and show a diffuse polymorphous infiltrate that includes a variable proportion of medium-sized neoplastic $T$ cells with abundant clear cytoplasm. these common characteristics in morphology often messed up with NPTCL$\mathrm{TFH}$, only through immunohistochemistry and molecular features can separate them[21-23]. With the new criteria, about 70\% AITL can be redefined as NPTCL-TFH[21, 22]. AITL and TFH-NPTCL, may represent a morphologic continuum is further strengthened by the reported observations of transitions from one diagnosis to the other[2, 12,23]. Our case's morphological feature is similar to AITL, but the exact expression of CD4, Bcl-6, PD-1 and CXCL-13, scattered EBV positive cells and the T cell receptor arrangement positive proved it $\mathrm{T}$ follicular helper cell phenotype. 
PTCL-NOS is another rare T-cell lymphoma needing to be distinguished. As the most common subtype of mature $T$ cell lymphoma, it is a diagnosis of exclusion. There is no particular morphological aspect of this tumor[24]. The lymph node often consists of numerous medium-sized or large cells with irregular, pleomorphic, hyperchromatic, or vesicular nuclei. Clear cells and Reed-Sternberg-like cells can also be seen. Unlike PTCL-TFH, PTCL-NOS has a very important inflammatory background in morphology, while hyperplasia of high endothelial venules and/or follicular dendritic cells are not usually seen[1]. Although our case displays similar cell feature with PTCL-NOS, it does not have a prominent polymorphic inflammatory background, and the expression of TFH markers instead of CD30, a possible marker of PTCL-NOS also supports our diagnosis.

Moreover, follicular T cell lymphoma (FTCL) has some similar features with PTCL-TFH, because they both derived from T follicular helper cells. Sang Eun Yoon et al. classified 207 cases diagnosed with nodal lymphomas of $T$ follicular helper (Tfh) cell origin into 111 cases of AITL, 68 cases of PTCL-NOS, 19 cases of FTCL and 10 cases of PTCL-TFH phenotype, but they cannot analyze FTCL and PTCL-TFH as different diseases with immunolabeling[25]. In FTCL, two distinct growth patterns are recognized: one that mimics follicular lymphoma and one that mimics progressive transformation of germinal canters[14]. The interfollicular areas lack the polymorphic infiltrates and vascular proliferation characteristic of AITL[26]. Our case shows no FDC expansion on CD21, which highlights the diagnose is not FTCL.

We did the immunohistochemical labeling of CD30 and ALK was to make a differential diagnosis with anaplastic large cell lymphoma(ALCL). ALCL can be divided into ALK-positive and ALK-negative subtypes based on expression of ALK or ALK gene rearrangement[1]. The neoplastic cells were mainly medium to large size with moderate basophilic cytoplasm, which mimics with our case. However, most of them had hyperchromatic nuclei and prominent nucleoli[27]. So added the negative expression of CD30 and ALK, we eliminate the consideration of ALCL.

Since some of the tumor cells were CD20 positive, we should not ignore some B-cell neoplasms such as Hodgkin lymphoma, High-grade B-cell lymphoma. It's just that the positive T cell markers and TCR rearrangement in our case confirmed that it was derived from T cells. Mantle T-cell lymphoma or other non-Hodgkin lymphomas such as Hepatosplenic T-cell lymphoma, Primary cutaneous anaplastic lymphoma, Primary cutaneous peripheral T-cell lymphomas also have some distinguishment points in our case. However, in general, it's the molecular feature, expression of Bcl-6, CD4, PD-1 and CXCL-13 and positive of TCR arrangement that confirms our diagnose.

\section{Conclusions}

More and more cases will be recognized with the growing knowledge of NPTCL-TFH. To do this correct diagnosis, we need to be aware that before diagnose a PTCL-NOS or AITL, use T follicular cell markers to eliminate NPTCL-TFH. Although very rare, CD20 can occur in NPTCL-TFH, exhaustive molecular and phenotypic investigations should be performed to get further understanding. 


\section{Abbreviations}

NPTCL-TFH, Nodal peripheral T-cell lymphoma with T follicular helper phenotype; AITL, Angioimmunoblastic T-cell lymphoma; PTCL-NOS, peripheral T-cell lymphoma, not other specified; FTCL, follicular T cell lymphoma; FDC, follicular dendritic cell; HEV, high endothelial venule; IHC, immunohistochemistry; EBER, Epstein-Barr virus (EBV)-encoded small RNA; HL, Hodgkin's lymphoma; $\mathrm{ALCL}$, anaplastic large cell lymphoma.

\section{Declarations}

\section{Consent}

The patient gave his written informed consents prior to inclusion in the study.

\section{Competing interests}

The authors declare that they have no competing interests.

\section{Authors' contributions}

$\mathrm{JH}$ and QC made the final diagnosis, $\mathrm{JH}$ and FL drafted the manuscript and performed the literature review; QC and FL contributed to concept, approved the final version of the manuscript. NL carried out the immunohistochemical examination. WZ collected the clinical data. All authors read and approved the final manuscript.

\section{Funding}

This work was supported by National Natural Science Foundation of China (Grant No. 81802431 and 81972516) and Natural Science Foundation of Guangdong Province (Grant No. 2018 A030313050 and 2021A1515012448). All these agencies have no roles in study design, data collection and analysis, and manuscript preparation

\section{References}

1. Swerdlow SHCE, Harris NL, Jaffe ES, Pileri SA,Stein H, Thiele J, Vardiman JW, editors: World Health Organization Classification of Tumours of the Haematopoietic and Lymphoid Tissues. IARC 2017:403-412.

2. Attygalle AD, Cabecadas J, Gaulard P, Jaffe ES, de Jong D, Ko YH, Said J, Klapper W. Peripheral T-cell and NK-cell lymphomas and their mimics; taking a step forward - report on the lymphoma workshop of the XVIth meeting of the European Association for Haematopathology and the Society for Hematopathology. Histopathology. 2014;64(2):171-99.

3. Vroobel KM, O'Connor S, Cunningham D, Wren D, Sharma B, Wotherspoon A, Attygalle AD. Florid T follicular helper cell hyperplasia associated with extranodal marginal zone lymphoma: a diagnostic 
pitfall which may mimic T cell lymphoma. Histopathology. 2019;75(2):287-90.

4. Siaghani PJ, Song JY. Updates of Peripheral T Cell Lymphomas Based on the 2017 WHO Classification. Curr Hematol Malig Rep. 2018;13(1):25-36.

5. Ghione P, Faruque P, Mehta-Shah N, Seshan V, Ozkaya N, Bhaskar S, Yeung J, Spinner MA, Lunning M, Inghirami $G$, et al. $T$ follicular helper phenotype predicts response to histone deacetylase inhibitors in relapsed/refractory peripheral T-cell lymphoma. Blood advances. 2020;4(19):4640-7.

6. Kuek LE, Leffler M, Mackay GA, Hulett MD: The MS4A family: counting past 1, 2 and 3. Immunology and Cell Biology 2016, 94(1):11-23.

7. Klasener K, Jellusova J, Andrieux G, Salzer U, Bohler C, Steiner SN, Albinus JB, Cavallari M, Suss B, Voll RE, et al: CD20 as a gatekeeper of the resting state of human B cells. Proc Natl Acad Sci U S A 2021, 118(7).

8. Kuijpers TW, Bende RJ, Baars PA, Grummels A, Derks IAM, Dolman KM, Beaumont T, Tedder TF, van Noesel CJM, Eldering E, et al. CD20 deficiency in humans results in impaired T cell-independent antibody responses. Journal of Clinical Investigation. 2010;120(1):214-22.

9. Armitage JO, Gascoyne RD, Lunning MA, Cavalli F. Non-Hodgkin lymphoma. Lancet. 2017;390(10091):298-310.

10. Ma CS, Deenick EK. Human T follicular helper (Tfh) cells and disease. Immunol Cell Biol. 2014;92(1):64-71.

11. Sandell RF, Boddicker RL, Feldman AL. Genetic Landscape and Classification of Peripheral T Cell Lymphomas. Current Oncology Reports 2017, 19(4).

12. Basha BM, Bryant SC, Rech KL, Feldman AL, Vrana JA, Shi M, Reed KA, King RL. Application of a 5 Marker Panel to the Routine Diagnosis of Peripheral T-Cell Lymphoma With T-Follicular Helper Phenotype. Am J Surg Pathol. 2019;43(9):1282-90.

13. Siaghani PJ, Wong JT, Chan J, Weisenburger DD, Song JY. Epidemiology and Pathology of T- and NKCell Lymphomas. Cancer treatment research. 2019;176:1-29.

14. Huang Y, Moreau A, Dupuis J, Streubel B, Petit B, Le Gouill S, Martin-Garcia N, Copie-Bergman C, Gaillard F, Qubaja M, et al. Peripheral T-cell Lymphomas With a Follicular Growth Pattern are Derived From Follicular Helper T Cells (T-FH) and may Show Overlapping Features With Angioimmunoblastic T-cell Lymphomas. Am J Surg Pathol. 2009;33(5):682-90.

15. Lemonnier F, Couronne L, Parrens M, Jais J-P, Travert M, Lamant L, Tournillac O, Rousset T, Fabiani B, Cairns RA, et al. Recurrent TET2 mutations in peripheral T-cell lymphomas correlate with T-FH-like features and adverse clinical parameters. Blood. 2012;120(7):1466-9.

16. Yokose N, Ogata K, Sugisaki Y, Mori S, Yamada T, An E, Dan K. CD20-positive T cell leukemia/lymphoma: case report and review of the literature. Ann Hematol. 2001;80(6):372-5.

17. Tachibana T, Tomita N, Furuya M, Yamanaka S, Takeuchi K, Nakamura N, Fujita H, Ishigatsubo Y. Aberrant CD20 Expression in Angioimmunoblastic T-cell Lymphoma. Intern Med. 2011;50(5):495-9. 
18. Foukas PG, Kefala M, Papageorgiou S, Tsirigotis P, Panayiotidis P, Korkolopoulou P, Spathis A, Dervenoulas J, Patsouris E, Karakitsos P, et al. CD20 expression in angioimmunoblastic T cell lymphoma. Leuk Lymphoma. 2012;53(2):345-7.

19. Banz Y, Krasniqi F, Dirnhofer S, Tzankov A. Relapsed angioimmunoblastic T-cell lymphoma with acquired expression of CD20: a case report and review of the literature. BMC clinical pathology. 2013;13:18-8.

20. Wallin EF, Jolly EC, Suchanek O, Bradley JA, Espeli M, Jayne DRW, Linterman MA, Smith KGC. Human T-follicular helper and T-follicular regulatory cell maintenance is independent of germinal centers. Blood. 2014;124(17):2666-74.

21. Yao W-Q, Wu F, Zhang W, Chuang S-S, Thompson JS, Chen Z, Zhang S-W, Clipson A, Wang M, Liu H, et al. Angioimmunoblastic T-cell lymphoma contains multiple clonal T-cell populations derived from a common TET2 mutant progenitor cell. J Pathol. 2020;250(3):346-57.

22. Lemonnier F, Mak TW. Angioimmunoblastic T-cell lymphoma: more than a disease of T follicular helper cells. J Pathol. 2017;242(4):387-90.

23. Attygalle AD, Chuang SS, Diss TC, Du MQ, Isaacson PG, Dogan A. Distinguishing angioimmunoblastic T-cell lymphoma from peripheral T-cell lymphoma, unspecified, using morphology, immunophenotype and molecular genetics. Histopathology. 2007;50(4):498-508.

24. Jha KK, Gupta SK, Saluja H, Subedi N. Peripheral T-cell lymphoma, not otherwise specified. J Family Med Prim Care. 2017;6(2):427-30.

25. Yoon SE, Cho J, Kim YJ, Ko YH, Park WY, Kim SJ, Kim WS. Comprehensive analysis of clinical, pathological, and genomic characteristics of follicular helper T-cell derived lymphomas. Exp Hematol Oncol. 2021;10(1):9.

26. Ohshima K. Pathological features of diseases associated with human T-cell leukemia virus type I. Cancer Sci. 2007;98(6):772-8.

27. Cao QH, Liu F, Li SR, Liu N, Li LH, Li CZ, Peng TS. Primary rare anaplastic large cell lymphoma, ALK positive in small intestine: case report and review of the literature. Diagnostic Pathology 2016, 11.

\section{Figures}

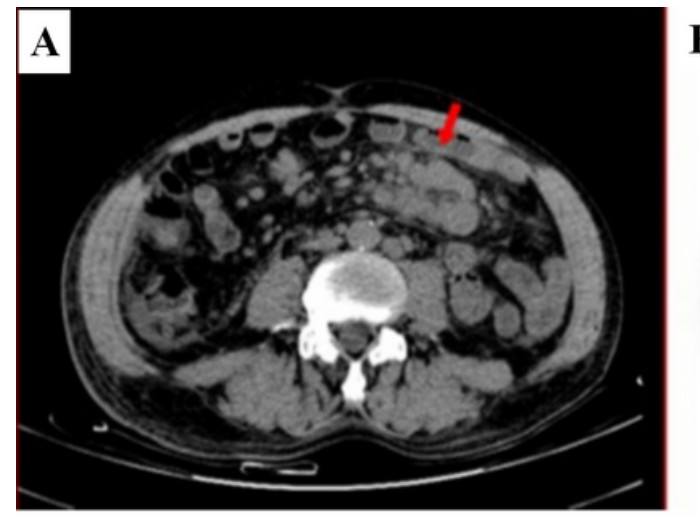

B
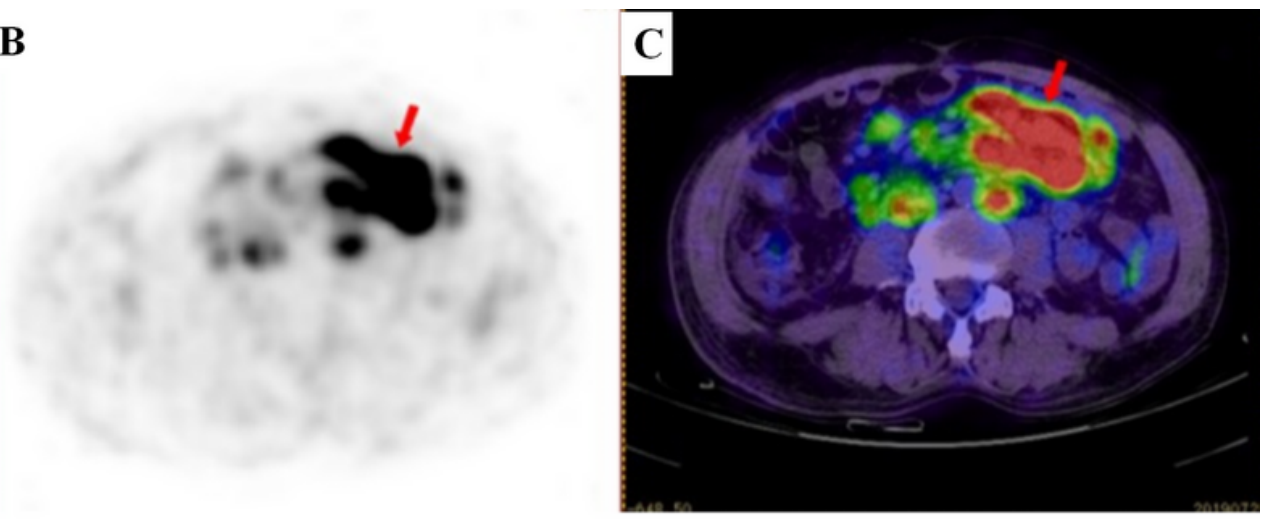


\section{Figure 1}

Positron emission tomography / computed tomography(PET/CT) images of the patient with NPTCL-TFH. A $3.4 \mathrm{~cm} \times 2.3 \mathrm{~cm}$ lymphadenopathy in abdomen. (A) Abdominal computed tomography image. (B) Positron emission tomography image. (C) merge of them. The red arrow indicate the lymphadenopathy.
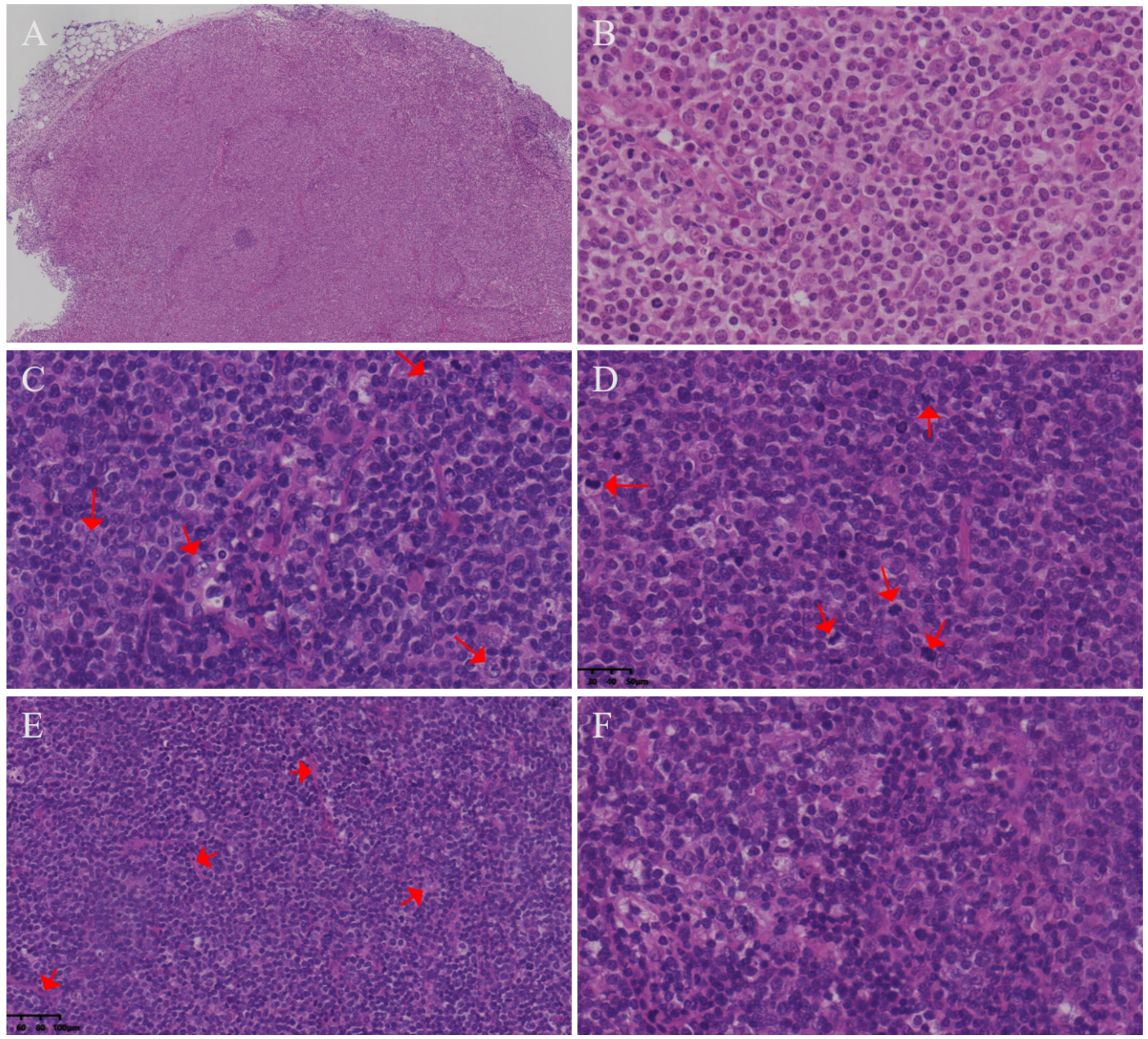

\section{Figure 2}

Hematoxylin and eosin(H\&E) stained sections of tumor: (A) Tumorx 40 showing the destruction of lymph nodes; (B) Tumorx 400 showing the relatively consistent tumor cells; (C) Tumor $\times 400$ showing large cells like immunoblast in AITL, the red arrow indicate the large cells; (D) Tumorx 400 showing frequently mitosis, the red arrow indicate the mitosis; (E) Tumor $\times 200$ showing vessels proliferation but few HEV 
proliferation, the red arrow indicate the vessels; (F) Tumorx 400 showing lack of inflammatory/polymorphous background.
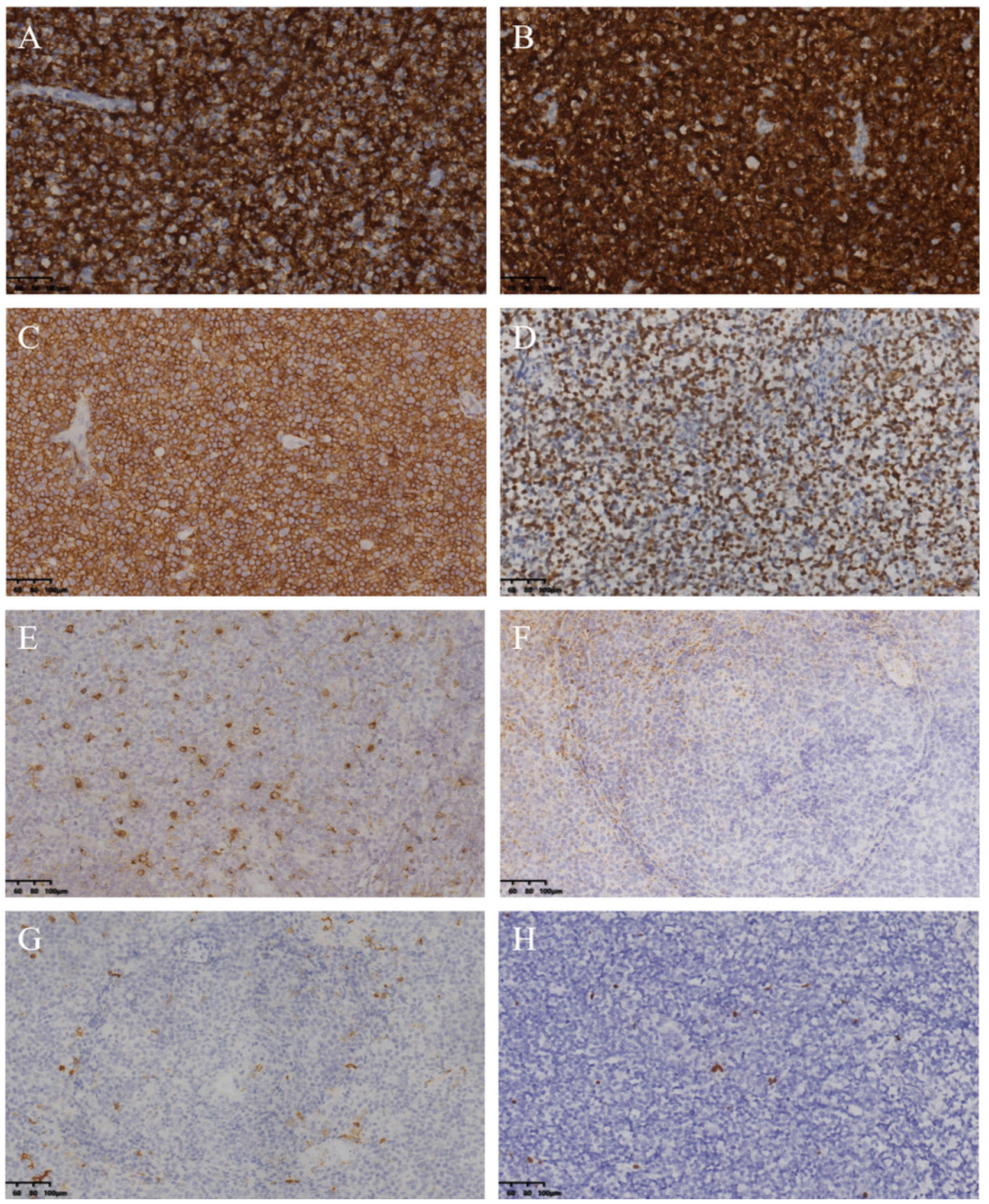

\section{Figure 3}

Immunohistochemical staining and in situ hybridization of PTCL-TFH. CD20(A), CD3(B) highlighting the lymphoid cells. CD4(C), Bcl-6(D) and CXCL-13(E) as the markers of TFH cell, shows positive. CD21(F) and 
CD23(G) shows no expansion of FDC. Scattered cells also present in situ hybridization of EBER(H) positive.

\section{Supplementary Files}

This is a list of supplementary files associated with this preprint. Click to download.

- CAREchecklistEnglish2013.pdf 